\title{
Scope for a small circumsolar annular gravitational contribution to the Pioneer anomaly without affecting planetary orbits
}

\author{
Guy S.M. Moore · Richard E.M. Moore
}

Received: 2 February 2013 / Accepted: 28 May 2013 / Published online: 14 June 2013

(C) The Author(s) 2013. This article is published with open access at Springerlink.com

\begin{abstract}
All proposed gravitational explanations of the Pioneer anomaly must crucially face the Equivalence Principle. Thus, if Pioneers 10 and 11 were influenced by anomalous gravitational effects in regions containing other Solar System bodies, then those bodies should likewise be influenced, irrespective of their shape, composition or mass. Although the lack of any observed influence upon planetary orbits severely constrains such explanations, here we aim to construct by computer modeling, hypothetical gravitating annuli having no gravitational impact on planetary orbits from Mercury to Neptune. One model has a central zone, free of radial gravitation in the annular plane, and an 'onset' beyond Saturn's orbit, where sunward annular gravitation increases to match the Pioneer anomaly data. Sharp nulls are included so that Uranus and Neptune escape this influence. Such models can be proportionately reduced in mass: a $1 \%$ contribution to the anomaly requires an annulus of approximately 1 Earth mass. It is thus possible to comply with the JPL assessment of newly recovered data attributing $80 \%$, or more, of the anomaly to spacecraft heat, which appears to allow small contributions from other causes. Following the possibility of an increasing Kuiper belt density at great ranges, another model makes an outward small anomalous gravitation in the TNO region, tallying with an observed slight indication of such an effect, suggesting that New Horizons may slightly accelerate in this region.
\end{abstract}

G.S.M. Moore $(\varangle) \cdot$ R.E.M. Moore

9 West Avenue, Sandown, Isle of Wight, PO36 9LT, UK

e-mail: gsmm.physics@gmail.com

R.E.M. Moore

e-mail: remm.physics@gmail.com
Keywords Experimental tests of gravitational theories . Lunar, planetary, and deep space probes · Orbit determination and improvement

\section{Nomenclature}

$r \quad$ heliocentric radius in the annular plane

$\sigma(r)$ annular surface mass density function

$g(r)$ sunward radial gravitation function in the annular plane

\section{Introduction}

Over the last decade, many stones have been turned in the effort to explain the Pioneer anomaly, from normal to new physics (reviewed by Turyshev and Toth 2010). The anomaly is an unexplained blueshift drift in radio-metric tracking data, interpreted as a small constant sunward acceleration of $(8.74 \pm 1.33) \times 10^{-10} \mathrm{~m} \mathrm{~s}^{-2}$ acting on Pioneer 10 and Pioneer 11 at distances of 20-70 AU (Turyshev et al. 2005). Quite separately, during the last decade, the evidence for the abundance of exoplanets and circumstellar disks containing gaps and rings gradually became very prominent, helping to place our Solar System into its evolutionary context (Meyer et al. 2007; Moro-Martin et al. 2008; Weinberger 2008; Moro-Martin 2013). Extensive exoKuiper belts around Sun-like stars are also being seen (Nilsson et al. 2010; Marshall et al. 2011; Donaldson et al. 2012) and modeled in preparation for further observations (Ertel et al. 2012). This perhaps suggests the importance today of looking more deeply into the physical possibilities lying beneath one particular stone, which we believe was first lifted in relation to the Pioneer anomaly, by de Diego et al. (2006). This concerns gravitating annuli much larger in radial extent and mass than the known characteristics of the 
Kuiper belt. The proposal was regarded as unlikely at that time (Nieto 2005; Bertolami and Vieira 2006), and a Kuiper belt model with a mass of $1 M_{\oplus}$, greater than standard estimates, had already been found to produce neither the constancy nor the magnitude of the forces necessary to explain the anomaly (Anderson et al. 2002a). Adding to the unlikelihood of the proposal is the Equivalence Principle, implying (Fienga et al. 2009), "if the equivalence principle is followed, the equations of motion of the major planets of our solar system have also to be modified in the same manner as the spacecraft dynamical equations are". This constraint also applies to modifications of the known laws of gravity which give a radial extra-force "in the region in which the Pioneer anomaly manifested itself in its presently known form" (Iorio 2009a).

The very notable lack of effect of the Pioneer anomaly upon planetary ephemerides has led to substantial constraints on any proposed gravitational explanations. For example, studying the orbits of Uranus, Neptune and Pluto, no unknown gravitational forces were found in the region 20$40 \mathrm{AU}$ (Iorio and Giudice 2006) or in the region of Saturn and Jupiter (Iorio 2007a) and it became "more and more difficult to realistically consider the possibility that some modifications of the current laws of Newton-Einstein gravity may be the cause of the Pioneer anomaly" (Iorio 2007b). No Pioneer anomaly forces were detectable in the motions of the moons of Neptune (Iorio 2010). Anderson et al. (1998) describe how explanations for the Pioneer anomaly involving dark matter or modifications of gravity "come up against a hard experimental wall" consisting of the NASA Viking range measurements of that time indicating that the Pioneer effect "is too large to have gone undetected in planetary orbits, particularly for Earth and Mars", and "would cause inconsistencies with the overall planetary ephemeris." Given that planetary ephemerides for the outer planets were less accurately known than for the inner planets, Tangen (2007) considered if there was scope for explaining the Pioneer anomaly either by an unknown distribution of matter in the outer Solar System or by spherically symmetric weak-field gravitational metric models, giving long range gravity modifications. However, the Equivalence Principle would imply that in all such cases the spacecraft would move geodesically, but this could not be matched to the available data. Rathke and Izzo (2006) concluded that if the effect of a Pioneer anomalous acceleration is parameterized in a change of effective reduced solar mass, then the effects on Neptune and Uranus would be an order of magnitude, or two, greater than the current observational constraints and "the anomaly exceeds by five orders of magnitude the corrections to Newtonian motion predicted by general relativity (at $50 \mathrm{AU}$ solar distance)." Today the constraints on anomalous accelerations for Earth and Mars are much tighter (Iorio 2009b;
Folkner 2010) and the current status of researches on general relativity in the Solar System is described by Iorio et al. (2011).

In spite of so much evidence against gravitational explanations of the Pioneer anomaly, we found a Newtonian method of bypassing the planetary constraints on gravitational explanations of the Pioneer anomaly imposed by the Equivalence Principle. Here our goal consists in ensuring that gravitating annular models make sunward radial gravitation only between and beyond the outer planets where the Pioneer anomaly was manifest, whilst producing zero radial gravitation for every planet, from Mercury to Neptune (although we investigate some precessional effects on Saturn in Sect. 4.3). In 2008, the importance of thermal recoil effects on Pioneer 10 and Pioneer 11 (discussed in Sect. 2) had not been realized, so we started exploring annular gravitation as if it might explain the entire Pioneer anomaly. It appeared then as if there was only one correct solution on a menu of many possibilities; for example, the coincidence of the anomalous acceleration with the Hubble acceleration had suggested a full solution in terms of cosmological expansion, a proposal ruled out by Lämmerzahl et al. (2006). Page et al. (2006) proposed using asteroids to study the anomaly and "once and for all, to either support or refute its existence as a real phenomenon" whereas we now argue for a multiplicity of small contributory causes, with thermal recoil from spacecraft heat being the greatest contributor. Their work on trans-Neptunian objects (TNOs) is considered in a potentially very interesting context in Sect. 5 .

Unaware of the work of de Diego et al. (2006), we searched for $\sigma(r)$ functions, able to make $g(r)$ functions containing a 'plateau', like in Fig. 1(a). A similar 'plateau' occurs in the work of de Diego et al. (2006), but in Fig. 1(a), we use more complicated empirical equations which include a convex knife-edge in the $\sigma(r)$ function, preventing a 'hump' from forming in $g(r)$ at the rim. The possibility of a truncation of the protoplanetary solar disk by a passing star (Moro-Martin et al. 2008), which could sustain $g(r)$ towards the truncation, looked very promising to us for explaining why the Pioneer anomaly maintained its strength at increasing distance from the Sun, suggesting the presence of an annular rim and in Fig. 1(a) the $g(r)$ 'plateau' more than covered the range of 20-70 AU where the Pioneer anomaly was apparent (Turyshev et al. 2005). We thought that the negative $g(r)$ in Fig. 1(a) might be hidden by spacecraft launching errors and trajectory corrections-spacecraft destined for planetary encounters were launched with additional errors so that the launch rocket didn't crash into the destination planet and cause possible biological contamination (Melbourne 1976). However, a brief discussion with Professor Carl Murray after his lecture on Saturn's rings to the Vectis Astronomy Society (June 27, 2008) sent our research in a new direction, lasting several years-he said that 


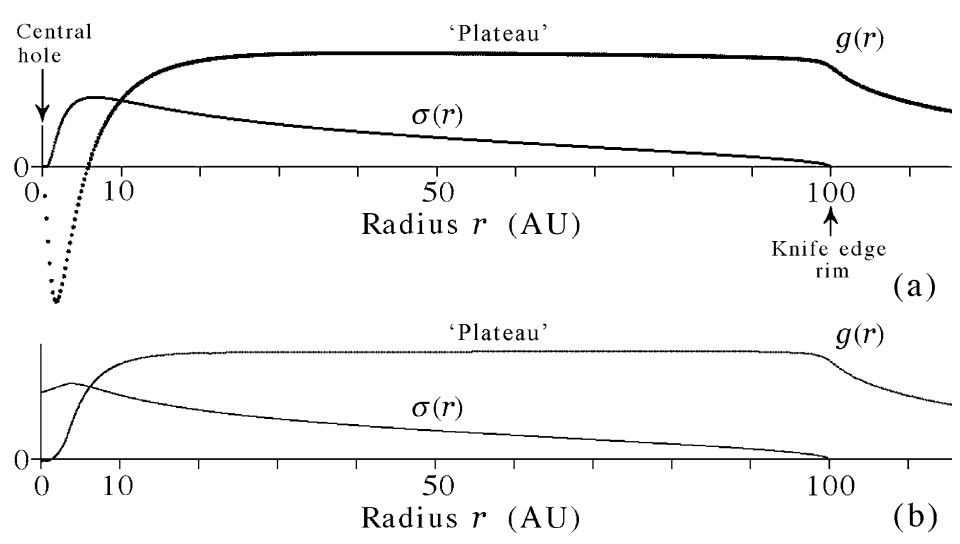

Fig. 1 (a) Our early attempt to make a plateau in the sunward radial gravitation function $g(r)$, with an annulus modeled by an empirical equation specifying the surface mass density function $\sigma(r)$, where $r$ is the heliocentric radius. (b) Filling the central hole by using a particular tangent to the peak of $\sigma(r)$ seen in (a), found by trial and error, produced the beginnings of a central neutral radial gravitational zone, from 0 to $2 \mathrm{AU}$, where $g(r)$ is close to zero. Mass calibration is discussed in the text an annulus of the type we describe would influence planetary orbits.

We then deduced by orbital modeling that the annulus of Fig. 1(a) would cause Mercury to precess prograde by an additional one-seventh of that caused by General Relativity. Thereafter we aimed to reduce the gravitational effect upon Mercury by shaping the $\sigma(r)$ function. Thus in Fig. 1(b), a $g(r)$ curve with the beginnings of a neutral zone was obtained, extending towards Venus, Earth and Mars. Striving to extend this neutral zone, rather than using empirical equations, it was easier to use graphical methods and curve adjusting tools described in the Appendix, to generate the required $\sigma(r)$ function. We aimed to reach to beyond Saturn's orbit (see particularly Fig. 6), eventually finding that the entire planetary Solar system could be covered. To illustrate this perhaps surprising annular effect and the essential mechanics, in Sect. 3 an annulus with a neutral zone extending to $90 \mathrm{AU}$ is modeled. But we do not discuss the plausibility or implausibility of such models until Sect. 6, having demonstrated in Sects. 4 and 5, their ability to match the Pioneer anomaly data.

A serious problem for annuli making accelerations equal to the Pioneer anomaly is their high masses. Setting the $g(r)$ 'plateau' in Fig. 1(a) equal to the Pioneer anomaly interpreted as a sunward acceleration of $(8.74 \pm 1.33) \times$ $10^{-10} \mathrm{~m} \mathrm{~s}^{-2}$ (Turyshev et al. 2005) fixes the scales giving $(240 \pm 36) M_{\oplus}$ for the annular mass. This is three orders of magnitude greater than the upper limit of $0.3 M_{\oplus}$ for the Kuiper belt dust discussed in Turyshev and Toth (2010), and many orders of magnitude greater than the $10^{16}$ to $10^{17} \mathrm{~kg}$ of cometary dust residue responsible for the Zodiacal light (Kopal 1979). We puzzled over this lack of observed mass because an annulus obeying Newton's laws of gravitation explaining the Pioneer anomaly represents a very attractive conventional solution for making forces pointing towards the Sun, but without the Sun being the direct gravitational cause. It has been discussed that if the direction of the Pioneer anomalous acceleration could be established more precisely, then this would help to establish possible causes-if towards Earth then this suggests a signal propagation effect or time signal anomaly, if along the spin axis then an onboard systematic, if along the velocity vector then an inertial or drag force, or if towards the Sun then a force "originating from the Sun, likely signifying a need for gravity modification" (Turyshev and Toth 2009, 2010). But it seemed possible that a gravitating annulus could obviate the need for gravity modification in the outer Solar System. The $306 M_{\oplus}$ obtained by de Diego et al. (2006) for the mass of their annulus was discussed in terms of dark matter, but their annuli appear not to have been developed so as to leave planetary orbits unaffected. De Diego (2008) discusses other possibilities for explaining the anomaly and the necessity of ruling out on-board causes, and the results of further analysis and data recovery (Toth and Turyshev 2008) were then awaited. The significance of spacecraft thermal recoil forces then became apparent.

\section{Spacecraft thermal effects}

During our annular modeling, the picture concerning the importance of spacecraft systematics radically changed. Assessing spacecraft systematics is complex (Nieto and Anderson 2007; Turyshev and Toth 2010). Several evaluations (Anderson et al. 1998, 2002a, 2002b; Turyshev et al. 1999, $2005)$ indicated that the anisotropic radiation of spacecraft heat could only account for a small fraction of the anomalous acceleration, but later Turyshev and Toth (2010) say, "the magnitude of the recoil force due to anisotropically emitted heat has been underestimated". 
Estimations using Lambertian point modeling of the Pioneer spacecraft yielding a $35 \%$ to $65 \%$ contribution, were given by Bertolami et al. (2008), but uncertainties arise from the unknown effects of degradation and damage during such a long spaceflight upon the properties of spacecraft materials (Bertolami et al. 2010). Finite element modeling methods by Rievers et al. (2009, 2010, 2011) give an analysis which "likely resolves the anomalous acceleration observed for $\mathrm{Pi}$ oneer 10". When the whole anomaly could be explained (Bertolami et al. 2012; Bertolami and Páramos 2012), the Pioneer anomaly becomes "not of strong interest anymore" (Selig et al. 2012). But the JPL analysis of the newly recovered data (Turyshev et al. 2012) is more cautious: 80\% of the anomalous acceleration was most certainly caused by the anisotropic radiation of spacecraft heat, the remaining $\sim 20 \%$ appeared not statistically significant. This, too, may also be caused by thermal radiation but this appears uncertain.

Francisco et al. (2012) could account for "between $44 \%$ and $96 \%$ " of the anomaly, recognizing the uncertainties caused by spacecraft surface degradation, hence other causes are still possible but, "unless new data arises, the puzzle of the anomalous acceleration of the Pioneer probes can finally be put to rest". However such a high proportion of the anomaly being caused by heat was regarded as unlikely by the Principal Investigator in Celestial Mechanics at JPL for these spacecraft (Shiga 2011), and investigations have continued particularly concerning how a gravitational phenomenon might influence spacecraft but not planetary orbits (Anderson and Morris 2012a, 2012b). Spacecraft systematics is an obvious explanation, but other effects can do this too, e.g. 'Nonlinear Electrodynamics' (Mbelek et al. 2007), 'Conformal Cosmology' (Varieschi 2012), or an explanation based on differences between astronomical and atomic times (Rañada and Tiemblo 2009). A different method of analyzing the interaction between spacecraft spin and circular signal polarization, suggested its greater significance (Mbelek and Michalski 2004) but it "still remains to explain" the constant part of the Pioneer anomaly. Concluding, although the thermal contribution is able to explain the entire anomaly, this contribution has not been measured with the accuracy needed to rule out a variety of possible contributions from other physical effects. A classic case of missing an opportunity for discovery concerns Neptune-it would have been discovered fifty years earlier if one of its recorded positions had not been attributed to an error (Flammarion and Danjon 1964).

\section{An annular example where $g(r)=0$ covers a large central zone}

Purely from the point of view of illustrating principles by hypothetical example, an annulus is modeled in Fig. 2(a), centred on the Sun. It has the property that over the radius range 0-90 AU, at all points in the annular plane, inward and outward radial gravitation exactly balance, so $g(r)=0$ in this region, forming a 'neutral zone', an effect which we have not seen in the literature. We discovered that annuli could exhibit this effect by studying the phenomenon of the Pioneer anomaly, particularly the 'onset' discussed in Sect. 4.

This annulus with an 'onset' radius of $90 \mathrm{AU}$ and $g(r)$ rising to a 'plateau' was constructed using the $\sigma(r)$ curve adjusting algorithms of the Appendix. ${ }^{1}$ If such an annulus were to exist in the ecliptic plane of the Solar System, it would have very little gravitational effect upon planetary orbits, but the planets would need to be 'traveling through' the annulus, perhaps sweeping out a clear space within a bilamellate structure parallel to its plane (considered further in Sect. 6). Of course not all the planets are in the ecliptic plane with mathematical precision, so there may be slight radial components arising from the axial gravitation of such an annulus but the axial gravitation is likely to be very small, being zero on the annular plane. The chief point made here is that such an annulus could be very difficult to detect gravitationally because of its very small perturbing effect upon planetary orbits. In contrast to this, in Fig. 2(b), all the annulus is removed, apart from leaving a ring of matter in the Kuiper belt region, when it then becomes apparent from the resulting $g(r)$ function that this ring would then produce gravitational effects upon all the planets. This would normally enable a constraint on the mass of the ring to be obtained, but by embedding the ring within the rest of the annulus, its radial gravitational effects are nullified. It is these Newtonian annular gravitational effects which we utilize here.

We call the very large annulus in Fig. 2(a) a 'background' annulus, in the sense that it has very little gravitational influence upon Solar System bodies or spacecraft on trajectories near to its plane, and in an idealized coplanar situation, independently of its mass. Of course, the influence of such an annulus would become gravitationally detectable, in its plane, at radii greater than its 'onset' radius, hence our interest in New Horizons, discussed later. The annulus of Fig. 2(a) produces zero Pioneer anomalous acceleration out to $90 \mathrm{AU}$, but now we use this type of annulus to match the anomaly.

\section{Matching the Pioneer anomaly data}

Using the curve adjusting algorithms described in Appendix we found that it was possible, as in Fig. 3, to match accurately all the Pioneer anomaly data points obtained from

\footnotetext{
${ }^{1}$ So far we have been unable to derive analytical equations for such $\sigma(r)$ functions.
} 
Fig. 2 (a) An annulus with $\sigma(r)$ adjusted to make a neutral radial gravitation zone, where $g(r)=0$ from the center to 90 AU. (b) When all the annular material, apart from between 40 and $45 \mathrm{AU}$, is removed, then large gravitational effects appear within what was the neutral zone of (a). The vertical scales are identical for both pictures

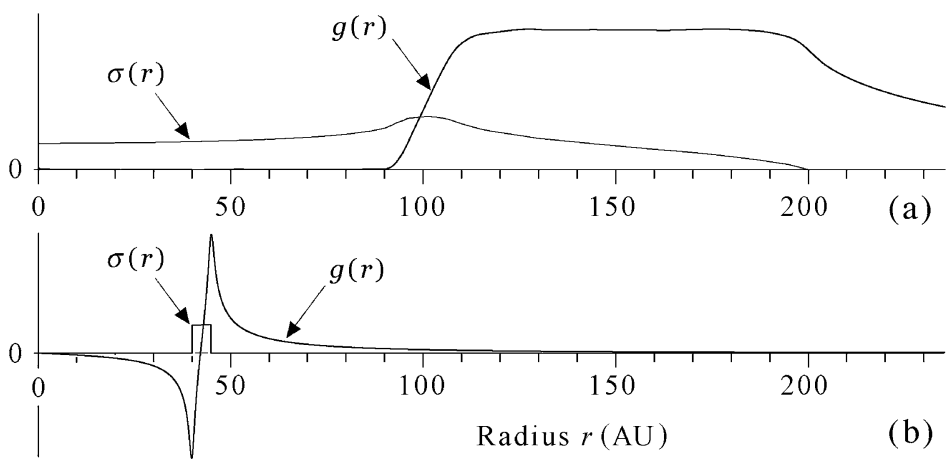

(b)

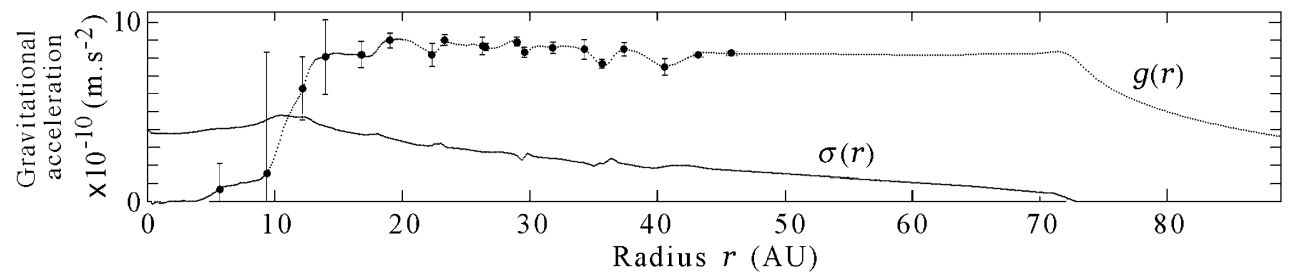

Fig. 3 A circumsolar annular model $\left(132 M_{\oplus}\right)$ giving a $g(r)$ function matching the Pioneer anomaly data points (Nieto and Anderson 2005, Table 2). The $g(r)$ 'plateau' continues to $70 \mathrm{AU}$, thus covering the range $20-70 \mathrm{AU}$ where the anomaly was "unambiguously present" (Dittus et al. 2005). Calibrated $\sigma(r)$ scales for this type of data-match are given in Figs. 4 and 5(a)
Table 2 of Nieto and Anderson (2005), as if caused entirely by the gravitational effects of an annulus. The frequently published JPL Orbital Data Program plot (Anderson et al. 2002a; Nieto and Anderson 2005, 2007; Nieto 2008; Turyshev and Toth 2010) of the early unmodeled accelerations of the spacecraft, suggested an 'onset' of the anomaly near to Saturn, but it was not known if this was related to the transition to hyperbolic orbit (Nieto and Anderson 2007), was caused by incorrect solar pressure calibration (Toth and Turyshev 2008; Turyshev and Toth 2010), or represented "a hint about the real cause of the Pioneer anomaly" (Toth and Turyshev 2008). This hint led us to design the types of annuli we describe here, such as in Fig. 3, where radial gravitation is adjusted to be zero from the center of the annulus out to a radius of several AU. This tallies with the Viking ranging data of that time, limiting any unmodeled accelerations acting on Earth and Mars to less than $0.1 \times 10^{-10} \mathrm{~m} \mathrm{~s}^{-2}$ (Anderson et al. 1998), a constraint which is now several orders of magnitude tighter (Iorio 2009b; Folkner 2010). Given the sizes of the error bars for the first two data points plotted in Fig. 3 it would be reasonable to adjust $g(r)$ to be zero from the Sun to beyond the orbital radius of Saturn.

\subsection{Inserting gravitational nulls}

We found that there was just enough room in between the Pioneer anomaly data points plotted in Fig. 3 to incorporate nulls so that $g(r)=0$ at the respective orbital radii of Uranus and Neptune. This yielded the annulus of Fig. 4, containing gaps and rings, making a sunward directed gravitational field in the regions between the outer planets and beyond, capable of influencing spacecraft, but without affecting the planets themselves. The $g(r)$ null set to a radius of $30.2 \mathrm{AU}$ in Fig. 4, corresponds to the orbital radius of Neptune which scarcely changed during the passage of Pioneer 11 across its wake. Likewise, the $g(r)$ null at $19.1 \mathrm{AU}$, is close to the orbital radius of Uranus for when Pioneer 11 passed by. (Pioneer 10 traveled on the opposite side of the Solar System.) With Neptune orbiting within the outer null, then no constraint applies to the annular mass, acting via the Principle of Equivalence, with respect to this planet. Uranus has a less circular orbit, but in principle an elliptically matching gap and ring could nullify annular gravitation around its entire orbit, but here we only illustrate, using axisymmetric modeling and simple computing, the basic mechanics of reducing the constraint of the Equivalence Principle upon the mass of a circumsolar annulus with respect to as many planets as possible.

The suggestion made here is that the gravitational nulls corresponding to the planetary orbits of Uranus and Neptune were sufficiently narrow that they did not show up in the Pioneer anomaly data, but there is an additional consideration: the $g(r)$ 'plateau' falls in strength only slowly with distance above and below the annular plane, whereas gravitational nulls 'fill in' more rapidly, so spacecraft, such as Pioneer 10 and Pioneer 11, flying a few to several AU from the orbital planes of Uranus and Neptune, could experience the constancy of a very small sunward annular gravitational effect without encountering planetary nulls. Using data from http://cohoweb.gsfc.nasa.gov/helios/heli.html, 


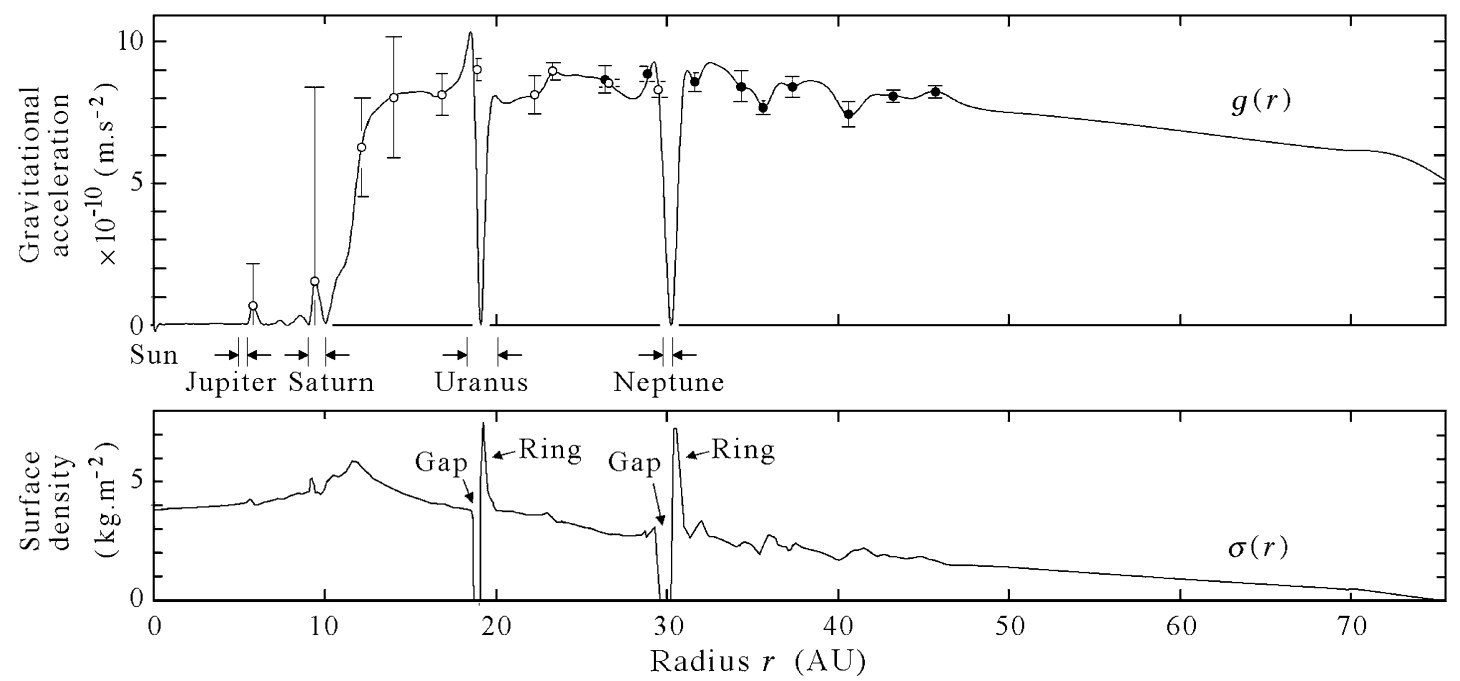

Fig. 4 A circumsolar annular model $\left(103 M_{\oplus}\right)$ matching the Pioneer anomaly data (Nieto and Anderson 2005, Table 2) including two outer planetary $g(r)$ nulls and a linearly decreasing $g(r)$ function over 50-70 AU (see Sect. 4.2). The upper graph contains the Pioneer 10 data points $(\bullet)$ and Pioneer 11 data points (o), including the tabulated

our estimated 'clearance distances' between Pioneer 11 and the orbital paths of Uranus and Neptune are $5 \mathrm{AU}$ and $8 \mathrm{AU}$ respectively, and for Pioneer 10, 1.1 AU and 2.5 AU respectively. At the closest orbital crossing, viz. Pioneer 10 crossing Uranus's orbit, the $g(r)$ null has already filled in to a level of $75 \%$, whereas at the farthest orbital crossing, viz. Pioneer 11 crossing Neptune's orbit, the $g(r)$ 'plateau' still operates at $70 \%$ of its full strength. Thus the Pioneers could have missed the planetary nulls because what remained of them on their particular trajectories, was hidden in the uncertainties.

\subsection{Matching the report of a decreasing anomalous acceleration}

The recent study (Turyshev et al. 2011), using newly recovered data, reported evidence that the Pioneer anomaly was decreasing slowly over time at $1.7 \times 10^{-11} \mathrm{~m} \mathrm{~s}^{-2} \mathrm{yr}^{-1}$ suggesting an association with the anisotropic radiation of spacecraft heat, very slowly decreasing, arising from the radioisotope thermoelectric generators. This effect can also be simulated by contouring the annulus, as in Fig. 4, so that $g(r)$ decreases at the spatial rate of $6.6 \times 10^{-12} \mathrm{~m} \mathrm{~s}^{-2} \mathrm{AU}$ ${ }^{-1}$ over the range 50-70 AU, and with Pioneer 10 traveling outwards here at $\sim 2.6 \mathrm{AU} \mathrm{yr}^{-1}$, the craft would then also experience an anomalous acceleration falling temporally at $1.7 \times 10^{-11} \mathrm{~m} \mathrm{~s}^{-2} \mathrm{yr}^{-1}$. With two possibilities for explaining the same effect, then a minor fractional contribution from annular gravitation could be masked by the major fractional contribution to the anomaly arising from anisotropically radiated spacecraft heat. error bar data, connected in their sequence of increasing radius by the $g(r)$ function, computed from the empirically adjusted $\sigma(r)$ function of the lower graph (see Appendix). The fluctuations in $g(r)$ up to the first two data points are discussed in Sect. 4.3. Planetary radial motions are indicated beneath the upper graph

\subsection{Uncertainties of the data near to Saturn and Jupiter}

There was considerable uncertainty (Nieto and Anderson 2005; Nieto 2008) of the data point near to Saturn, so there may well be a negligible Pioneer anomalous effect at this radius, particularly a gravitational one, especially since the constraints on this, discussed shortly, are now very tight. However, in Fig. 4, by adjusting $\sigma(r)$-but only as an example of what can be achieved with this type of annulus-we arbitrarily made nulls in the $g(r)$ function at the extremes of Saturn's radial motion (9.02 AU perihelion, 10.05 AU aphelion) preserving this data point 'intact' in between. Given that this annular gravitation, when superimposed upon solar gravity, would cause a slight departure from Newton's inverse square law, we used simple orbital modeling to investigate the consequent perihelion precession of Saturn caused by this annulus - it could be made prograde or retrograde, depending upon the shape of the $g(r)$ curve between aphelion and perihelion radii. By making fine adjustments to the $\sigma(r)$ function, we found it possible to hold the precession of Saturn to within \pm 0.02 arcsec of zero precession per orbital cycle, being at the limit of accuracy of our orbital modeling. Saturn's retrograde precession is very small, $-0.006 \pm 0.002^{\prime \prime} \mathrm{cy}^{-1}$, but is difficult to explain (Iorio $2009 \mathrm{c})^{2}$

\footnotetext{
${ }^{2}$ We determined that the mass of a pair of elemental rings, spaced $1 \mathrm{AU}$ above and below Saturn's orbital plane respectively, with heliocentric radius equal to the mean orbital radius of Saturn, required to explain the observed retrograde precession, is $\sim 0.00004 M_{\oplus}$. (Our modeling indicated that coplanar heliocentric rings either smaller than Saturn's
} 
Fig. 5 (a) A larger annulus $\left(144 M_{\oplus}\right)$ matching the Pioneer anomaly data (Nieto and Anderson 2005, Table 2), with $g(r)$ decreasing linearly, similar to Fig. 4. In (b) the annulus seen in (a) has the surface density reduced to $0.2 \sigma(r)$ over the range $0-40 \mathrm{AU}$, with no change over 55-120 AU, the two portions of curve are connected by half a cycle of inverted cosine curve over 40-55 AU.

The $g(r)$ function is computed from the $\sigma(r)$ function in each respective picture

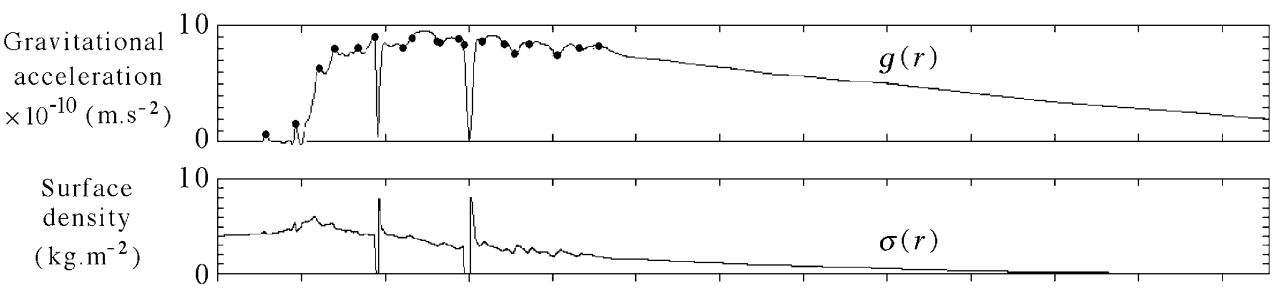

(a)

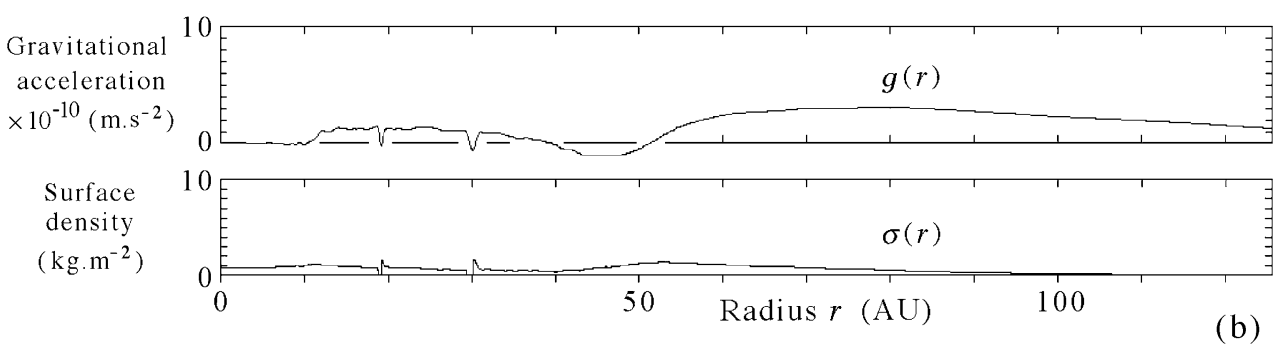

We made the central neutral gravitational zone in Fig. 4 extend from the Sun to Jupiter's aphelion (5.46 AU), thus exempting Jupiter from gravitational influence by this annulus. This is followed by a fluctuation up to the first detection of the anomaly at 5.80 AU, but the uncertainties in the first two Pioneer anomaly data points are large and it appears very certain (Iorio 2007a) that any significant gravitational Pioneer anomalous effects cannot begin until beyond Saturn's orbit. The Cassini mission has set tighter limits with respect to the ephemeris of Saturn (Standish 2009; Iorio 2012a), but the modeling described here would allow Saturn to orbit within a $g(r)$ neutral zone. We determined that even with Saturn's precession adjusted to as near zero as possible in models like Fig. 4, the orbital period of Saturn would be reduced by some twenty-one minutes, so there is much to suggest that only an exceedingly small anomalous gravitational effect could exist at Saturn, and this is easily modeled with this type of annulus.

\section{Effects in the far solar system}

The Pioneer data can be matched by larger annular models which can be adjusted to produce less of a contribution to the anomaly, as well as to model gravitational effects in the far Solar System. We begin with the annulus of mass $144 M_{\oplus}$ in Fig. 5(a) matching the Pioneer anomaly data, having $g(r)$ likewise decreasing linearly as the annulus of Fig. 4 starting at $50 \mathrm{AU}$, but extending to $120 \mathrm{AU}$.

Stern (1996) discusses the possibility of a 'trough' in the surface density of the Kuiper belt, with an increasing circumsolar disk density beyond $\sim 50 \mathrm{AU}$, where the disk "may even approach the primordial surface mass density". Teplitz

perihelion radius, or greater than Saturn's aphelion radius both cause prograde precession respectively.) et al. (1999) discuss a two sector Kuiper belt model with "a more distant sector with a higher density". In a Solar System analogue, the star LkCa 15 has "an outer disk that begins at $50 \mathrm{AU}$ " and an inner disk of uncertain size where planets are believed to be forming (Currie and Grady 2012).

A higher surface density of matter in the far Kuiper belt region could generate significant radial gravitation, and we tentatively model this in Fig. 5(b) by reducing the $\sigma(r)$ function of Fig. 5(a), to one-fifth (it could be less) over the range 0-40 AU, with a smooth curved transition to the value of $\sigma(r)$ of Fig. 5(a) at 55 AU, with no change beyond. The $g(r)$ function then makes a $\sim 20 \%$ contribution to the Pioneer anomaly out to $30 \mathrm{AU}$, containing a central neutral zone and outer planetary nulls as previously, as well as tallying with the maximum allowable $\sim 20 \%$ proportion of the anomaly which could be attributed to causes other than heat emission in the recent JPL analysis (Turyshev et al. 2012). Beyond $30 \mathrm{AU}$ the $g(r)$ function gradually falls to zero, going negative (i.e. acts outwards) over 40-50 AU, followed by a substantial increase beyond $50 \mathrm{AU}$.

Studies of trans-Neptunian objects (TNOs), mostly in the region 30-50 AU, gave an outward acting anomalous gravitation of $(-0.87 \pm 1.6) \times 10^{-10} \mathrm{~m} \mathrm{~s}^{-2}$, i.e. one-tenth the magnitude of the Pioneer anomalous acceleration, but "consistent with zero" because of the large uncertainties (Wallin et al. 2007). However, their data can also be interpreted as consistent with the type of annulus seen in Fig. 5(b), perhaps giving an observational hint of more mass in the far Kuiper belt, which would tally with such a possibility (Stern 1996). We are unsure what values to use for $\sigma(r)$ at such large ranges: a curve (Brown 2004, Fig. 2) suggests $10 \mathrm{~kg} \mathrm{~m}^{-2}$ at $80 \mathrm{AU}$, whereas Stern's figure at $80 \mathrm{AU}$ is $0.04 \mathrm{~kg} \mathrm{~m}^{-2}$ (Stern 1996, Fig. 5). We use an 'in between value' of $0.5 \mathrm{~kg} \mathrm{~m}^{-2}$ at $80 \mathrm{AU}$, in Fig. 5(b), giving rise to slight radial forces away from the Sun prior to the increase 
in surface mass density, so the $g(r)$ function, beyond $50 \mathrm{AU}$, may be several times greater or smaller than indicated.

This might be of concern to Oort cloud and long-period comet modeling because in the far Solar System where comets are more easily perturbed (Masi et al. 2009), anomalous gravitational effects, like Rindler gravitation or similar, operating at $\sim 10 \%$ of the Pioneer anomalous acceleration, could cause large departures from Keplerian orbits (Iorio 2012b). Moreover annular gravitation has cylindrical symmetry rather than the spherical symmetry assumed for a Pioneer anomalous acceleration in the studies of its possible effects (at full strength) upon comets by Whitmire and Matese (2003).

In the two sector Kuiper belt model of Teplitz et al. (1999), discussed above, the nearer sector (40-70 AU) has an estimated mass of $13 M_{\oplus}$ regarded as "unlikely", whereas a similar mass for its far sector (70-120 AU) is more sustainable because "If the far sector is dynamically cool enough that collisions are adhesive rather than fragmenting, there is little dust and no real IR limit on sector mass". The model of Fig. 5(b) has masses of $10 M_{\oplus}$ for the near sector and $6 M_{\oplus}$ for the far sector, and given all the approximations, this is in reasonable agreement with this two sector model; but this is a preplanetary Kuiper belt model. Nevertheless, a consideration has been made that the present day Kuiper belt rises in surface density to the primordial value, discussed above (Stern 1996). High values for mass in the Kuiper belt region of the present day, compared to the fractions of $0.1 M_{\oplus}$ found by studying precessional data for Mercury, Venus, Earth and Mars (Iorio 2007c), could only be gravitationally sustainable if the Kuiper belt formed a part of a greater annulus making near zero radial gravitation in the inner planetary region. But this is less of a constraint if the mass is located much further away in a belt extending to hundreds of $\mathrm{AU}$, like the exo-Kuiper belts beginning to be observed around other Sun-like stars (Nilsson et al. 2010; Marshall et al. 2011; Donaldson et al. 2012).

\section{Discussion}

We note that with respect to the Kuiper belt, there is a "missing-mass" problem (Chiang et al. 2007) viz. the primordial and present-day masses "differ by 2 orders of magnitude", and early studies by Stern suggested "the Kuiper belt did not even contain enough mass to have formed itself" (Brown 2004). Levison et al. (2007) summarize some problems: the value of $0.1 M_{\oplus}$ for the Kuiper belt mass "is surprising given that accretion models predict that $\geq 10 M_{\oplus}$ must have existed in this region in order for the objects that we see to grow" and if the migration of Neptune stopped at $30 \mathrm{AU}$, "how did the Kuiper belt lose $>99 \%$ of its mass?" The inferred initial masses for some debris disks around
Sun-like stars appear to be "surprisingly high" (Shannon and $\mathrm{Wu}$ 2011). This suggests that the mass estimations of the Kuiper belt seem to be too low, and if realistic possibilities were found for increasing the mass estimations of the Kuiper belt, then they merit further consideration. In this respect the statement, quoted in Sect. 5, concerning the far sector of a Kuiper belt model where infrared measurements place "no real IR limit on sector mass" (Teplitz et al. 1999) appears significant, and in relation to exo-Kuiper belt dust mass estimation, submillimeter thermal radiation gives "just a lower limit" (Nilsson et al. 2010).

The type of annuli described here widen the possibilities for Solar System modeling, allowing additional mass to be incorporated without upsetting planetary ephemerides. Since a 'mathematically thin' annulus on the scale of the Solar System may be several AU thick in places, then the height distribution of matter (from the annular plane) is another variable; it could be bilamellate with less detectable matter near to the ecliptic plane, apart from rings making $g(r)$ nulls which need to be close to the respective orbits of Uranus and Neptune. Concerning why planetary nulls may form physically, it is noted that exoplanets can open gaps and deplete matter on the insides of their orbits (Meyer et al. 2007; Moro-Martin et al. 2008; Weinberger 2008). This is in the correct direction to reduce the effect of annular gravitation with respect to the planet doing the shifting, but a mechanism is needed to explain why planetary orbits would tend to become near perfectly Keplerian. Departures from Keplerian orbits are associated with precession, and if precessional stirring of annular matter diminished, then orbital energy dissipation would also reduce and the Keplerian orbit would become more stable. But unless some physical mechanism like this operated, leading to only very slightly perturbed Keplerian orbits, then the very accurate Solar System ephemeris data (Pitjeva 2009) would be difficult to explain.

Standish (2009) tested various gravitational 'forms' for the Pioneer anomaly for their effects upon planetary ephemerides, finding that a constant sunward acceleration at the $10 \%$ level, could be ruled out and of the four radial-velocity dependent forms, only one generating forces beyond Saturn's orbit was compatible with planetary ephemerides. Iorio (2009a) also studied these gravitational 'forms' pointing out that they need sound theoretical justification. But it appears that annuli of the type described here with a neutral central zone and planetary nulls, or one having a neutral zone covering the entire planetary region of the Solar System, would pass such tests given that they have been modeled specifically so as not to influence planetary orbits.

We suggest, in connection with matter in the Solar System which so far may have eluded detection, that during the formation of planets with the depletion of gas and dust 
from the primordial circumsolar disk (Meyer et al. 2007; Moro-Martin et al. 2008; Weinberger 2008) that a less detectable form of matter may have remained in the Solar System. This might be permissible because the young subject of circumstellar disks is characterized by surprises, such as the high abundance of carbon being found in the disk of $\beta$ Pictoris (Weinberger 2008). We note that non-baryonic matter scarcely features, if at all, in the many papers on circumstellar disks in Reipurth et al. (2007), but certainly the 'flyby' anomaly (Nieto and Anderson 2009) suggests that unknown physical aspects of the Solar System have yet to be taken into account. Iorio (2006, 2013a) determined, from planetary orbital motions, upper bounds for a spherical dark matter density above the galactic background and these values give several orders of magnitude less than $1 M_{\oplus}$ for dark matter out to the orbital radius of Saturn. Edsjö and Peter (2010), taking into account an inverse process of ejection, indicate that the Solar System could only capture dark matter up to a fraction at most of the Galactic halo density.

If the contribution to the Pioneer anomaly of a gravitating annulus is reduced to $1 \%$, then using the data for the annulus of Fig. 4, its total mass would be reduced to $\sim 1 \mathrm{M}_{\oplus}$, spread over the range $0-70 \mathrm{AU}$, but a smooth distribution such as this in the inner Solar System, still seems problematic in comparison to the very small mass of the Zodiacal cloud. Over the range $0-1 \mathrm{AU}$ the annular mass is then $\sim 0.05 M_{\oplus}$, several orders of magnitude greater than the mass of the Zodiacal cloud. Small circumsolar rings quite close to the Sun have been proposed (see references in Iorio 2012c), but they are discrete rings, not smooth mass distributions, with masses many orders of magnitude less than $1 \mathrm{M}_{\oplus}$.

Even if $\sigma(r)$ is set to zero in the inner Solar System for our annular gravitating models, there is still a mathematically degenerate large variety of possible distributions of circumsolar disc matter, which in theory could extend to hundreds of AU, which would not exceed the constraint set by studies of the precession of the inner planets (Iorio 2007c), simply because the further this matter is away, the less its gravitational influence upon the planets. There is evidence that circumstellar discs can possess an outer ring extending to hundreds of $\mathrm{AU}$ beginning further out from an inner ring extending to tens of $\mathrm{AU}$ (Broekhoven-Fiene et al. 2012; Bonsor et al. 2013). This appears to have similarities to the structure suggested by Stern (1996) (Sect. 5), but if enough annular mass exists in outer structures to exceed the constraint set by the smallness of planetary precessions, then additional annular mass must exist in the inner Solar System to nullify the gravitational effects of the outer mass, following the mechanics of Sect. 3.

The fact is that uncertainties increase in the far Solar System, the Zodiacal light tends to screen the Kuiper belt (Moro-Martin et al. 2008; Moro-Martin 2013), the Sun's gravitation is less and so gravitational effects which have not so far been allowed for, may have increasing significance. Thus what happens to the New Horizons spacecraft becomes of increasing interest, particularly if $g(r)$ goes negative in the TNO region, followed by a substantial increase, as modeled in Fig. 5(b). It is important to understand gravitational effects in the Solar System to very high accuracy with respect to testing general relativity and fundamental physics (Iorio 2011; Iorio 2013b; Nobili et al. 2009) and tracking New Horizons is important with respect to constraining a possible massive trans-Plutonian object because of the gravitational effects this could have (Iorio 2013c).

\section{Summary and conclusions}

The Pioneer anomaly has stimulated many researches and here we explored the properties of gravitating annular models, finding that all the data could be accurately matched. We found how to generate a large central zone free of radial gravitation in the annular plane, with an 'onset', marking the beginning of a sunward radial gravitation field which can be set to beyond the orbital radius of Saturn. Beyond the 'onset', the field can be adjusted to be near constant, or to fall gradually, matching the falling Pioneer anomalous acceleration of recent reports. By incorporating gaps and rings into the annulus, nulls in the radial gravitation can be made to correspond with the orbits of Uranus and Neptune, thus liberating the mass of this annulus from the constraints of the Equivalence Principle with respect to all planetary orbits from Mercury to Neptune. An annular model, matching the Pioneer anomaly data, extending to $120 \mathrm{AU}$, was modified to comply with the recent JPL analysis of newly recovered data, and to model the possibility that in the far Kuiper belt, the surface density rises to primordial values. The annular model then generated slight outward forces in the TNO region, tallying with the possible indication that TNOs may experience very small such forces.

The weak point of these models is their high mass with no observational evidence for it, particularly in the inner Solar System which these models require in order for their radial gravitation to be manifest only in the outer Solar System. This is ameliorated by the Pioneer anomaly now being attributed mostly to spacecraft systematics, however, this has not been measured with sufficient accuracy to rule out perhaps as much as a $20 \%$ contribution from a multiplicity of other causes. This could be significant in terms of Solar System physics and might include annular gravitational effects too.

Although the validity of Newton's laws in the outer Solar System remains unconfirmed (Turyshev and Toth 2009) and support for the 'onset' is weak (Turyshev et al. 2011), a small Newtonian annular gravitational contribution to the Pioneer anomaly is here shown to be feasible and compatible with the Equivalence Principle. 
Acknowledgement We are very grateful for a detailed referee's report causing us to deal in greater depth with the literature and to improve our paper presentation. We thank the Frewen Library of Portsmouth University and the Public Libraries of the Isle of Wight.

Open Access This article is distributed under the terms of the Creative Commons Attribution License which permits any use, distribution, and reproduction in any medium, provided the original author(s) and the source are credited.

\section{Appendix: Computational methods}

\section{A.1 Annulus simulation}

We simulate a thin axisymmetric annulus with adjustable surface mass density $\sigma(r)$ by using a distribution of point masses placed at points in a network. The network is formed from a system of concentric circles in the $x y$-plane (i.e. the annular plane), centred on the origin, spaced by 0.01 $\mathrm{AU}$, intersecting radials spaced by $2^{\circ}$, starting and finishing at 1 arcdeg from the $x$-axis. (100 'computer program length units' $\equiv 1$ AU.) Half-way between the origin and the first circle, and between each pair of adjacent circles, point masses are placed on the radials to form rings, coequal on each ring, adjusted to the required $\sigma(r)$ function. This is the 'zeroth system' specification. When evaluating gravitation at a point $P$ in the annular plane, it is computationally slow to apply Newton's inverse square law to every mass point, so distant mass points are selected in groups along each radial and each group is treated as a single mass point. This is done by specifying the annulus at three lower resolutions, so the respective widths of the rings of each system are $0.01 \times 5^{\circ} \mathrm{AU}$, where $n=0,1,2$ or 3 and $n$ is the system label. For each system with $n=1,2$ or 3 , the 'replacement mass' placed at the mid-point on every radial between the inner and outer radii of each member ring, is the sum of the point masses of the zeroth system within this radius range. Radial gravity is computed at a point $P$, where a unit test mass is placed, incrementing along the $x$-axis in 0.05 AU steps, using Newton's inverse square law applied to every mass point in contiguous bands of rings selected from the four systems. When $P$ is distant from the origin, the band with $n=1$ extends 6 AU each side of $P$, then $n=2$ within 20 AU of $P$, and $n=3$ beyond, the precise boundaries are selected to give integer numbers of member rings per band. For $P$ closer than $2 \mathrm{AU}$ to the origin, $n=0$ is used from the origin out to the radius of $P$, and to $0.2 \mathrm{AU}$ beyond. These values are determined empirically and we obtain better than single-pixel accuracy when simulating an annulus of known mathematical properties (Moore and Moore 2009). In recent times we use modern programming software ${ }^{3}$ suitable for a PC but the computed gravita-

\footnotetext{
${ }^{3}$ Available from http://www.rtrussell.co.uk
}

tional properties of this precisely specified physical model are independent of the method of computation, provided it is accurately done.

\section{A.2 Calibration}

Scale calibration firstly assigns the MKS system of units to the computer program units of mass, length and time respectively, with Newton's gravitational constant $G$ set to unity. The resulting $g(r)$ data is then divided by the square of the ratio of the size of the annulus required 'in reality' to the size of the annulus in the computer program and $G$ is reset to $6.67 \times 10^{-11} \mathrm{~N} \mathrm{~kg}^{-2} \mathrm{~m}^{2}$. The annular mass is increased proportionately so that the $g(r)$ data, such as a 'plateau' or a data point used as a reference, then tallies with the $\mathrm{Pi}$ oneer anomalous acceleration or with the data point. This yields the annular mass and calibration for the $g(r)$ and $\sigma(r)$ scales.

\section{A.3 Curve adjusting algorithms}

Figure 6 illustrates how to generate an annulus with a central neutral $g(r)$ zone, beginning with only straight line portions to construct the $\sigma(r)$ function. The basic curve adjusting tools required are:-

1. An interpolation algorithm which fills in the values for $\sigma(r)$ linearly between $r_{1}$ and $r_{2}$, for specified values of $\sigma\left(r_{1}\right)$ and $\sigma\left(r_{2}\right)$. Thus in Fig. 6(a), $\sigma(0)$ is set to a constant and $\sigma\left(r_{\text {edge }}\right)$ is already zero (all values in the array for $\sigma(r)$ are initially zero), whence the entire $\sigma(r)$ function, a straight line, is constructed from the annular center to the annular edge, where $r=r_{\text {edge}}$. In Fig. 6(b), $\sigma(0)$ is reset to a lower value, and interpolated to the radius where the 'onset' in $g(r)$ is required.

2. A 'triangular pulling' algorithm, taking three points on the $\sigma(r)$ curve, at radii $r_{1}, r_{2}$ and $r_{3}$ respectively $\left(r_{1}<r_{2}<r_{3}\right)$, multiplies the value of $\sigma\left(r_{2}\right)$ by a chosen factor (close to unity for fine adjusting), and interpolates $\sigma(r)$ between $\sigma\left(r_{1}\right)$ and $\sigma\left(r_{2}\right)$, and between $\sigma\left(r_{2}\right)$ and $\sigma\left(r_{3}\right)$ respectively. This is used to thicken the annular rim in Fig. 6(b), and to generate the 'onset' in $g(r)$ in two stages in (c) and (d).

3. The 'zigzag' $\sigma(r)$ function of Fig. 7 is useful for pulling the $g(r)$ curve towards data points. It can have leading and trailing portions which multiply existing values of $\sigma(r)$ by small linear terms subtracted from and added to unity respectively (forming the 'outer portions' of the 'zigzag'), interpolating only across the central section. The chosen outer and central widths of the function, and its amplitude, depend on nearby data points, with empirical adjusting to give the best result. When inverted, this function raises a portion of the $g(r)$ curve. Application 
Fig. 6 An annulus with an approximate central neutral radial gravitation zone and an 'onset' is made in four stages using straight lines to form the $\sigma(r)$ function. In (a) the 'annulus blank', prior to shaping, has a linearly decreasing $\sigma(r)$, giving the corresponding computed $g(r)$. In (b) the rim is thickened by adding a thin triangular portion, and the inner part of $\sigma(r)$ is truncated. In (c) and (d) the $\sigma(r)$ function is pulled upwards in triangular fashion in two stages, forming the steep 'onset' in $g(r)$. The dashed lines in each picture correspond to the $\sigma(r)$ function of the previous picture. Further shaping makes a more accurate neutral central zone, a flatter or sloping 'plateau', and data points can be matched, accompanied by scale calibration. Figures 2-7 were constructed by these methods (a)

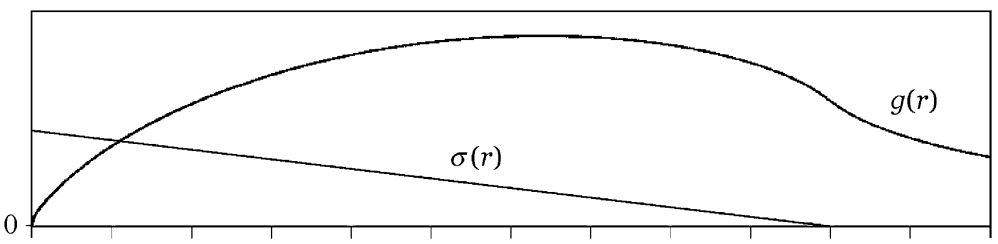

(b)

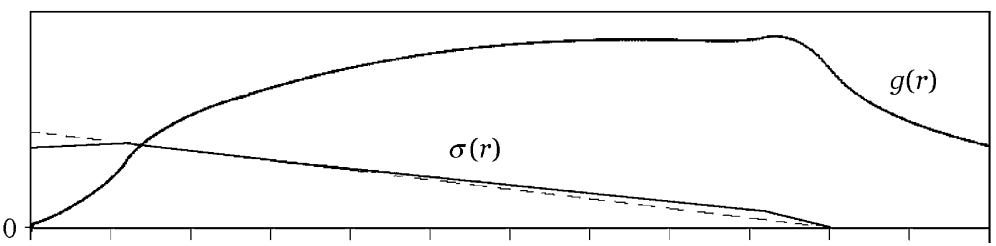

(c)

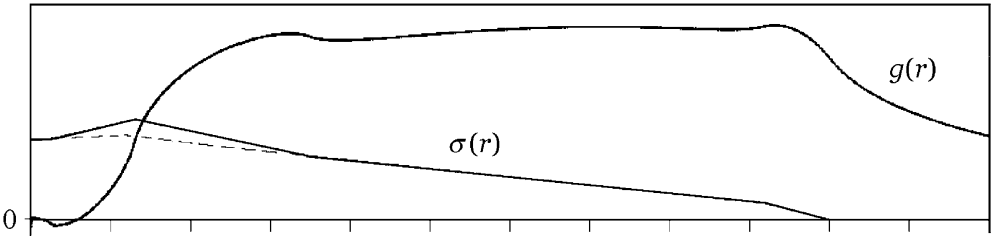

(d)

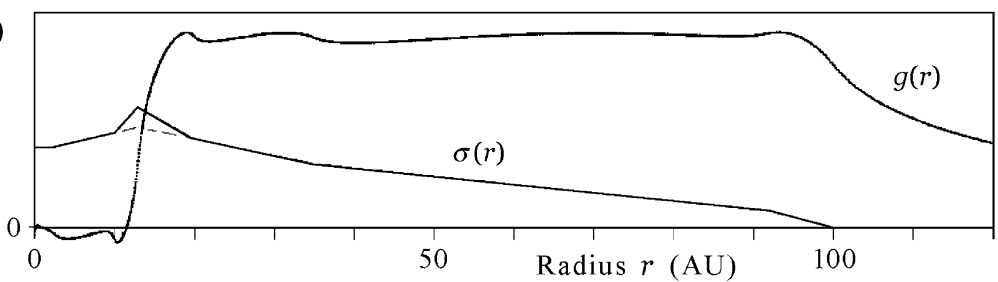

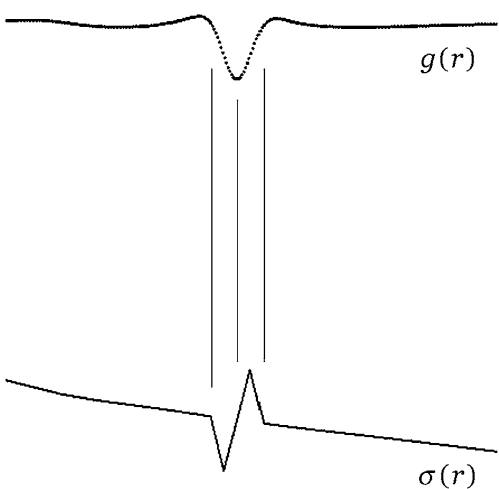

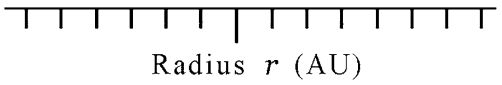

Fig. 7 The 'zigzag' function in $\sigma(r)$ causes a dip in $g(r)$, accompanied by small rises in $g(r)$ spread into each side, giving scarcely any change in mean $g(r)$. This function (and its inverse) is useful to obtain $g(r)$ curves matching the Pioneer anomaly data points, as in Figs. 3, 4 and 5(a). (Vertical scales uncalibrated)

of this function scarcely changes the total annular mass or the mean $g(r)$ in the region of the function.
4. Sometimes large sections of the $g(r)$ curve need slight vertical shifting. This can be done by multiplying longish sections of the $\sigma(r)$ curve, term by term, by expressions such as $\left\{1-0.005 \sin \left[\left(r-r_{1}\right) \pi /\left(r_{2}-r_{1}\right)\right]\right\}$ for $r_{1} \leq r \leq r_{2}$, or a similar expression using a one-quarter cycle of cosine function, starting at the origin and finishing at the 'onset' radius, and adjusting the parameters for the best result.

5. When adjusting the $\sigma(r)$ curve to make nulls in the $g(r)$ curve, an algorithm operating on one or both sides of the null, shifting the values of $\sigma(r)$ to larger or smaller radii, has application.

\section{References}

Anderson, J.D., Morris, J.R.: Phys. Rev. D 86(6), 064023 (2012a) Anderson, J.D., Morris, J.R.: Phys. Rev. D 85(8), 084017 (2012b)

Anderson, J.D., Laing, P.A., Lau, E.L., Liu, A.S., Nieto, M.M., Turyshev, S.G.: Phys. Rev. Lett. 81(14), 2858 (1998)

Anderson, J.D., Laing, P.A., Lau, E.L., Liu, A.S., Nieto, M.M., Turyshev, S.G.: Phys. Rev. D 65(8), 082004 (2002a)

Anderson, J.D., Lau, E.L., Turyshev, S.G., Laing, P.A., Nieto, M.M.: Mod. Phys. Lett. A 17(14), 875 (2002b)

Bertolami, O., Vieira, P.: Class. Quantum Gravity 23(14), 4625 (2006)

Bertolami, O., Páramos, J.: (2012). arXiv:1212.2177 [gr-qc]

Bertolami, O., Francisco, F., Gil, P.J.S., Páramos, J.: Phys. Rev. D 78(10), 103001 (2008) 
Bertolami, O., Francisco, F., Gil, P.J.S., Páramos, J.: Space Sci. Rev. 151(1-3), 75 (2010)

Bertolami, O., Francisco, F., Gil, P.J.S., Páramos, J.: (2012). arXiv:1211.6939 [gr-qc]

Bonsor, A., Kennedy, G.M., Crepp, J.R., Johnson, J.A., Wyatt, M.C., Sibthorpe, B., Su, K.Y.L.: (2013). arXiv:1302.7000 [astro-ph]

Broekhoven-Fiene, H., Matthews, B.C., Kennedy, G.M., Booth, M., Sibthorpe, B., Lawler, S.M., Kavelaars, J.J., Wyatt, M.C., Qi, C., Koning, A., Su, K.Y.L., Rieke, G.H., Wilner, D.J., Greaves, J.S.: Astrophys. J. 762(1), 52 (2012)

Brown, M.E.: Phys. Today 57(4), 49 (2004)

Chiang, E., Lithwick, Y., Murray-Clay, R., Buie, M., Grundy, W., Holman, M.: A brief history of trans-Neptunian space. In: Reipurth, B., Jewitt, D., Keil, K. (eds.) Protostars and Planets V, p. 895. University of Arizona Press, Tucson (2007)

Currie, T., Grady, C.: Sky Telesc. 124(2), 20 (2012)

de Diego, J.A., Núñez, D., Zavala, J.: Int. J. Mod. Phys. D 15(4), 533 (2006)

De Diego, J.A.: The anomalous acceleration of the Pioneer spacecrafts. Rev. Mex. Astron. Astrofís., Ser. Conf. 34, 35 (2008). arXiv:0807.0617 [physics.space-ph]

Dittus, H., Turyshev, S.G., Lämmerzahl, C., Theil, S., Foerstner, R., Johann, U., Ertmer, W., Rasel, E., Dachwald, B., Seboldt, W., Hehl, F.W., Kiefer, C., Blome, H.-J., Kunz, J., Giulini, D., Bingham, R., Kent, B., Sumner, T.J., Bertolami, O., Páramos, J., Rosales, J.L., Christophe, B., Foulon, B., Touboul, P., Bouyer, P., Reynaud, S., Brillet, A., Bondu, F., Samain, E., de Matos, C.J., Erd, C., Grenouilleau, J.C., Izzo, D., Rathke, A., Anderson, J.D., Asmar, S.W., Lau, E.L., Nieto, M.M., Mashhoon, B.: ESA Spec. Publ., vol. 588 p. 3 (2005)

Donaldson, J.K., Roberge, A., Chen, C.H., Augereau, J.-C., Dent, W.R.F., Eiroa, C., Krivov, A.V., Mathews, G.S., Meeus, G., Ménard, F., Riviere-Marichalar, P., Sandell, G.: Astrophys. J. 753(2), $12(2012)$

Edsjö, J., Peter, A.H.P.: (2010). arXiv:1004.5258 [astro-ph]

Ertel, S., Wolf, S., Rodman, J.: Astron. Astrophys. 544, A61 (2012)

Fienga, A., Laskar, J., Kuchynka, P., Leponcin-Lafitte, C., Manche, H., Gastineau, M.: (2009). arXiv:0906.3962 [gr-qc]

Flammarion, G.C., Danjon, A.: The Flammarion Book of Astronomy, p. 324. Allen \& Unwin, London (1964)

Folkner, W.M.: IAU Symp. 261, 155 (2010)

Francisco, F., Bertolami, O., Gil, P.J.S., Páramos, J.: Phys. Lett. B 711(5), 337 (2012)

Iorio, L.: J. Cosmol. Astropart. Phys. 5, 2 (2006)

Iorio, L.: J. Gravit. Phys. 1, 5 (2007a)

Iorio, L.: Found. Phys. 37(6), 897 (2007b)

Iorio, L.: Mon. Not. R. Astron. Soc. 375(4), 1311 (2007c)

Iorio, L.: Int. J. Mod. Phys. D 18(6), 947 (2009a)

Iorio, L.: Astrophys. Space Sci. 323(3), 215 (2009b)

Iorio, L.: Astron. J. 137(3), 3615 (2009c)

Iorio, L.: Mon. Not. R. Astron. Soc. 405(4), 2615 (2010)

Iorio, L.: Int. J. Mod. Phys. D 20(2), 181 (2011)

Iorio, L.: Mod. Phys. Lett. A 27(12), 1250071 (2012a)

Iorio, L.: Mon. Not. R. Astron. Soc. 419(3), 2226 (2012b)

Iorio, L.: Earth Moon Planets 108(3-4), 189 (2012c)

Iorio, L.: (2013a). arXiv:1304.6396 [gr-qc]

Iorio, L.: (2013b). arXiv:1302.6920 [gr-qc]

Iorio, L.: (2013c). arXiv:1301.3831 [gr-qc]

Iorio, L., Giudice, G.: New Astron. 11(8), 600 (2006)

Iorio, L., Lichtenegger, H.I.M., Ruggiero, M.L., Corda, C.: Astrophys. Space Sci. 331(2), 351 (2011)

Kopal, Z.: The Realm of the Terrestrial Planets, p. 170. Institute of Physics, Bristol (1979)

Lämmerzahl, C., Preuss, O., Dittus, H.: (2006). arXiv:gr-qc/0604052

Levison, H.F., Morbidelli, A., Gomes, R., Backman, D.: Planet migration in planetesimal disks. In: Reipurth, B., Jewitt, D., Keil, K. (eds.) Protostars and Planets V, p. 669. University of Arizona Press, Tucson (2007)
Marshall, J.P., Löhne, T., Montesinos, B., Krivov, A.V., Eiroa, C., Absil, O., Bryden, G., Maldonado, J., Mora, A., Sanz-Forcada, J., Ardila, D., Augereau, J.-C., Bayo, A., del Burgo, C., Danchi, W., Ertel, S., Fedele, D., Fridlund, M., Lebreton, J., González-Garcia, B.M., Liseau, R., Meeus, G., Müller, S., Pilbratt, G.L., Roberge, A., Stapelfeldt, K., Thébault, P., White, G.J., Wolf, S.: Astron. Astrophys. 529, A117 (2011)

Masi, M., Secco, L., Gonzalez, G.: Open Astron. J. 2, 74 (2009). arXiv:0911.5533 [astro-ph.GA]

Mbelek, J.P., Michalski, M.: Int. J. Mod. Phys. D 13(5), 865 (2004)

Mbelek, J.P., Mosquera Cuesta, H.J., Novello, M., Salim, J.M.: Europhys. Lett. 77(1), 19001 (2007). arXiv:astro-ph/0608538

Melbourne, W.G.: Sci. Am. 234(6), 58 (1976)

Meyer, M.R., Backman, D.E., Weinberger, A.J., Wyatt, M.C.: Evolution of circumsolar disks around normal stars: placing our solar system in context. In: Reipurth, B., Jewitt, D., Keil, K. (eds.) Protostars and Planets V, p. 573. University of Arizona Press, Tucson (2007)

Moore, G., Moore, R.: Math. Gaz. 93, 347 (2009)

Moro-Martin, A., Wyatt, M.C., Malhotra, R., Trilling, D.E.: Extrasolar Kuiper Belt dust disks. In: Barucci, M.A., Boehnhardt, H., Cruikshank, D.P., Morbidelli, A. (eds.) The Solar System Beyond Neptune, p. 465. University of Arizona Press, Tucson (2008)

Moro-Martin, A.: Dusty planetary systems. In: Oswalt, T.D., French, L.M., Kalas, P. (eds.): Planets, Stars and Stellar Systems, vol. 3, p. 431. Springer, Dordrecht (2013). arXiv:1203.0005 [astro-ph]

Nieto, M.M.: Phys. Rev. D 72(8), 083004 (2005)

Nieto, M.M.: Phys. Lett. B 659(3), 483 (2008)

Nieto, M.M., Anderson, J.D.: Class. Quantum Gravity 22(24), 5343 (2005)

Nieto, M.M., Anderson, J.D.: Contemp. Phys. 48(1), 41 (2007)

Nieto, M.M., Anderson, J.D.: Phys. Today 62(10), 76 (2009)

Nilsson, R., Liseau, R., Brandeker, A., Olofsson, G., Pilbratt, G.L., Risacher, C., Rodmann, J., Augereau, J.-C., Bergman, P., Eiroa, C., Fridlund, M., Thébault, P., White, G.J.: Astron. Astrophys. 518, A40 (2010)

Nobili, A.M., Comandi, G.L., Pegna, R., Bramanti, D., Doravari, S., Maccarone, F., Lucchesi, D.M.: IAU Symp. 261, 390 (2009)

Page, G.L., Dixon, D.S., Wallin, J.F.: Astrophys. J. 642(1), 606 (2006)

Pitjeva, E.V.: IAU Symp. 263, 93 (2009)

Rañada, A.F., Tiemblo, A.: (2009). arXiv:0909.0912 [gr-qc]

Rathke, A., Izzo, D.: J. Spacecr. Rockets 43(4), 806 (2006)

Reipurth, B., Jewitt, D., Keil, K. (eds.): Protostars and Planets V. University of Arizona Press, Tucson (2007)

Rievers, B., Bremer, S., List, M., Lämmerzahl, C., Dittus, H.: New J. Phys. 11(11), 113032 (2009)

Rievers, B., Bremer, S., List, M., Lämmerzahl, C., Dittus, H.: Acta Astron. 66(3), 467 (2010)

Rievers, B., Lämmerzahl, C.: Ann. Phys. 523(6), 439 (2011)

Selig, H., Lämmerzahl, C., Ni, W.-T.: (2012). arXiv:1212.3645 [gr-qc]

Shannon, A., Wu, Y.: Astrophys. J. 739(1), 36 (2011)

Shiga, D.: New Sci. 210(2807), 12 (2011)

Standish, E.M.: IAU Symp. 261, 179 (2009)

Stern, S.A.: Astron. J. 112, 1203 (1996)

Tangen, K.: Phys. Rev. D 76(4), 042005 (2007)

Teplitz, V.L., Stern, S.A., Anderson, J.D., Rosenbaum, D., Scalise, R.J., Wentzler, P.: Astrophys. J. 516(1), 425 (1999)

Toth, V.T., Turyshev, S.G.: AIP Conf. Proc. 977, 264 (2008)

Turyshev, S.G., Toth, V.T.: Space Sci. Rev. 148(1-4), 149 (2009)

Turyshev, S.G., Toth, V.T.: Living Rev. Relativ. 13(4), 9 (2010)

Turyshev, S.G., Anderson, J.D., Laing, P.A., Lau, E.L., Liu, A.S., Nieto, M.M.: (1999). arXiv:gr-qc/9903024

Turyshev, S.G., Nieto, M.M., Anderson, J.D.: Am. J. Phys. 73(11), 1033 (2005)

Turyshev, S.G., Toth, V.T., Ellis, J., Markwardt, C.B.: Phys. Rev. Lett. 107(8), 081103 (2011) 
Turyshev, S.G., Toth, V.T., Kinsella, G., Lee, S.-C., Lok, S.M., Ellis, J.: Phys. Rev. Lett. 108(24), 241101 (2012)

Varieschi, G.U.: Phys. Res. Int. 2012, 469095 (2012)

Wallin, J.F., Dixon, D.S., Page, G.L.: Astrophys. J. 666(2), 1296 (2007)
Weinberger, A.J.: Sky Telesc. 116(5), 32 (2008)

Whitmire, D.P., Matese, J.J.: Icarus 165(1), 219 (2003) 